\title{
Dominating points and large deviations for random vectors
}

\author{
U. Einmahl ${ }^{1, \star}$, J. Kuelbs ${ }^{2, \star \star}$ \\ ${ }^{1}$ Department of Mathematics, Indiana University, Bloomington, IN 47405, USA \\ ${ }^{2}$ Department of Mathematics, University of Wisconsin, Madison, WI 53706, USA
}

Received: 12 September 1995/In revised form: 13 December 1995

Summary. We establish a representation formula useful for obtaining precise large deviation probabilities for convex open subsets of a Banach space. These estimates are based on the existence of dominating points in this setting.

Mathematics Subject Classification (1991): 60B12, 60F10

\section{Introduction}

In [10], and later in [11], dominating points, and a representation formula useful for obtaining precise large deviation estimates for probabilities on $\mathbb{R}^{d}$ were obtained. The final section of the paper [1] initiated a similar study for the Banach space setting, and here we continue this line of investigation. We establish natural analogues of the results in [10] and [11], under the usual assumptions for large deviation probabilities in the infinite dimensional setting. Throughout we assume $B$ is a real separable Banach space with norm $\|\cdot\|$, Borel subsets $\mathscr{B}$, and topological dual denoted by $B^{*}$. $\mathbb{P}$ denotes the Borel probability measures on $(B, \mathscr{B})$.

If $\mu \in \mathbb{P}, f \in B^{*}$, then $\hat{\mu}(f)=\int_{B} e^{f(x)} d \mu(x)$ is the Laplace transform of $\mu$, and for $x \in B$ we define

$$
\lambda(x)=\sup _{g \in B^{*}}[g(x)-\log \hat{\mu}(g)] .
$$

Then $\lambda(\cdot)$ is the so-called rate function for $\mu$, and $\operatorname{dom}(\lambda)=\{x: \lambda(x)<$ $\infty\}$. We will assume throughout that $\mu \in \mathbb{P}$ is not concentrated at a single

Dedicated to Peter Ney on the occasion of his 65th birthday.

* Supported in part by NSF Grant DMS-9503665

$\star \star$ Supported in part by NSF Grant DMS-9400024 
point and is such that

$$
\int_{B} e^{t\|x\|} d \mu(x)<\infty
$$

for all $t>0$. The assumption (1.2) is standard in the infinite dimensional setting, but is a bit strong in $\mathbb{R}^{d}$. Our definition of a dominating point is as follows:

Definition. Let $\mu \in \mathbb{P}$. If $D$ is an open convex subset of $B$ with $D \cap \operatorname{dom}(\lambda) \neq \emptyset$, then $a_{0} \in \bar{D}$ is called a dominating point for $(D, \mu)$ if $a_{0} \in \partial D$, the boundary of $D$,

$$
\lambda\left(a_{0}\right)=\inf _{x \in D} \lambda(x)=\inf _{x \in \bar{D}} \lambda(x),
$$

and for some $g \in B^{*}$ we have $D \subseteq\left\{x: g(x) \geqq g\left(a_{0}\right)\right\}$ with

$$
\lambda\left(a_{0}\right)=g\left(a_{0}\right)-\log \hat{\mu}(g) .
$$

Theorem 1. Let $\mu \in \mathbb{P}$ have mean zero, and assume (1.2) holds. Let $D$ be an open convex subset of $B$ such that $0 \notin D$ and $D \cap \operatorname{dom}(\lambda) \neq \emptyset$. Then there exists a unique point $a_{0} \in \partial D$ such that $a_{0}$ is a dominating point for $(D, \mu)$. Furthermore, if $X, X_{1}, X_{2}, \ldots$ are i.i.d. with $\mu=\mathscr{L}(X)$ and $S_{n}=X_{1}+\cdots+X_{n}$ for $n \geqq 1$, then the following representation holds:

$$
P\left(S_{n} \in n D\right)=\exp \left\{-n \lambda\left(a_{0}\right)\right\} J_{n},
$$

where

$$
J_{n}=E\left[\exp \left(-g\left(Z_{1}+\cdots+Z_{n}\right)\right) I_{\left\{Z_{1}+\cdots+Z_{n} \in n\left(D-a_{0}\right)\right\}}\right],
$$

$g \in B^{*}$ satisfies (1.4), $D \subseteq\left\{x: g(x) \geqq g\left(a_{0}\right)\right\}$ and $Z, Z_{1}, Z_{2}, \ldots$ are i.i.d. mean zero random variables with $\mathscr{L}(Z)=\tilde{\gamma}$, where

$$
\tilde{\gamma}(d x)=\exp \left\{g(x)+g\left(a_{0}\right)\right\} \gamma(d x) / \hat{\mu}(g)
$$

with

$$
\gamma(\cdot)=\mathscr{L}\left(X-a_{0}\right) .
$$

Remarks. (1) The representation in (1.5) is the precise analogue of that in [10] and in [11] when $B=\mathbb{R}^{d}$, although our assumption (1.2) is stronger. However, in infinite dimensional spaces (1.2) is close to being best possible for large deviation probabilities for $S_{n}$. In addition, our proof is quite different, and proceeds without the use of differentiability.

(2) The main point of Theorem 1 is that a dominating point always exists under the conditions imposed on $D$, which are near best possible as can be seen from remark (3) below. In [1] a candidate for the dominating point $a_{0}$ of $(D, \mu)$ is obtained, but the condition (1.4) is not established except when $D$ is assumed to be flat at $a_{0}$. Our proof shows that (1.4) holds in general.

(3) Since $\mu$ has mean zero, the assumption $0 \notin D$ is necessary, or $\lim _{n} P\left(S_{n} / n \in\right.$ $D)=1$ by the law of large numbers. The assumption $D \cap \operatorname{dom}(\lambda) \neq \emptyset$ is also necessary since Lemma 2.4 of [3] implies that $\overline{c o(\operatorname{supp} \mu)}=\overline{\operatorname{dom}(\lambda)}$. Here 
$\operatorname{co}(A)$ denotes the convex hull of $A, \operatorname{supp} \mu$ is the topological support of $\mu$ on $(B, \mathscr{B})$, and as usual $\bar{A}$ is the closure of $A$.

(4) It is trivial to see that Theorem 1, as well as Theorems 2 and 3 below, can be formulated to apply to measures $\mu$ which have mean other than zero by simply applying them to the centered measure. However, there is a more subtle extension which also holds, and given as Theorem $1^{*}$ at the end of this section.

The proof of Theorem 1 follows from the results of Sect. 2, and is given in Sect. 3. In order to state Theorem 2 we now need the material of Sect. 2 as included in the following definition.

Definition. Let $D$ be a convex subset of $B$ with $0 \notin D$, and assume $a_{0}$ is the unique dominating point for $(D, \mu)$. If $a_{0} \neq 0$, take $f \in B^{*}$ such that (2.6) holds. We say $D$ contains slices whose diameters near $a_{0}$ dominate the function $\tau(s)=(s|\log s|)^{1 / 2}$ if there exists $a \in B, \delta>0, \beta>0$ such that $f(a)>f\left(a_{0}\right)$, and for $x_{0}=a-a_{0}, 0 \leqq s \leqq \delta$ and $M_{s}=\left\{x: f(x)=s f\left(x_{0}\right)\right\}$ we have

$$
M_{s} \cap\left(D-a_{0}\right) \supseteq\left\{y+s x_{0}: y \in M_{0},\|y\| \leqq \beta \tau(s)\right\} .
$$

If $a_{0}=0$, then we say $D$ contains slices whose diameters near zero dominate the function $\tau(s)=(s|\log s|)^{1 / 2}$ if there exists $f \in B^{*}, a \in B, \delta>0, \beta>0$ such that $D \subseteq\{x: f(x)>0\}, f(a)>0$, and (1.8) holds with $x_{0}=a$.

Now we are able to formulate some additional results.

Theorem 2. Let $\mu \in \mathbb{P}$ have mean zero, and assume (1.2) holds. Let $D$ be an open convex subset of $B$ with $0 \notin \bar{D}$ and such that $D \cap \operatorname{dom}(\lambda) \neq \phi$. If $a_{0}$ is the dominating point of $(D, \mu)$, then there exists a constant $C_{1}<\infty$ such that for all $n \geqq 1$

$$
P\left(S_{n} \in n D\right) \leqq C_{1} n^{-1 / 2} \exp \left\{-n \lambda\left(a_{0}\right)\right\} .
$$

Furthermore, if $\left\{T_{n} / \sqrt{n}\right\}$ is bounded in probability, where we set $T_{n}=Z_{1}+\cdots+Z_{n}, Z_{1}, Z_{2}, \ldots$ are as in Theorem 1 , and $D$ contains slices whose diameters near $a_{0}$ dominate the function $\tau(s)=(s|\log s|)^{1 / 2}$, then we also have $C_{2}>0$ such that

$$
P\left(S_{n} \in n D\right) \geqq C_{2} n^{-1 / 2} \exp \left\{-n \lambda\left(a_{0}\right)\right\} .
$$

Moreover, if $D$ only contains slices whose diameters near $a_{0}$ dominate the power function $s^{1 / 2}$, then there exists a finite constant $C_{3}$ such that

$$
P\left(S_{n} \in n D\right) \geqq n^{-1 / 2} \exp \left\{-C_{3}(\log n)^{1 / 2}\right\} \exp \left\{-n \lambda\left(a_{0}\right)\right\} .
$$

Remarks. If (1.8) holds when the function $\tau(s)$ is replaced by the power function $s^{1 /(1+\alpha)}$ for $\alpha>1$, then the slices near $a_{0}$ are even bigger (recall $0 \leqq$ $s \leqq \delta$ ) and hence (1.10) holds provided the other assumptions of Theorem 2 are valid. Moreover, if $D$ is a ball in a 2 -smooth space, then at every point of its boundary $D$ contains slices whose diameters dominate the power function $s^{1 / 2}$. (See [9] for details.) Finally, recall that if $B$ is a type 2 Banach space, 
then $\left\{T_{n} / \sqrt{n}\right\}$ is always bounded in probability, but in general this need not be the case.

If $B$ is a Hilbert space, then our next result shows that $\tau(s)$ can be replaced by $s^{1 / 2}$ in (1.10) so that we have in this case a better result than for general Banach spaces.

Theorem 3. Let $B$ be a Hilbert space with $\mu \in \mathbb{P}$ having mean zero and (1.2) holding. Let $D$ be an open convex subset of $H$ with $0 \notin \bar{D}$ and $D \cap \operatorname{dom}(\lambda) \neq \phi$. Let $a_{0}$ be the dominating point of $(D, \mu)$, and assume that $D$ contains slices whose diameters near $a_{0}$ dominate the power function $s^{1 / 2}$. Then (1.10) holds.

Remarks. Of course, the upper bound (1.9) holds as well, so up to differences in $C_{1}$ and $C_{2}$ we have the correct asymptotic behavior of $P\left(S_{n} \in n D\right)$ in Hilbert spaces. Also note that Theorem 3 includes all open balls $D$ in $H$ with $0 \notin \bar{D}$ and $D \cap \operatorname{dom}(\lambda) \neq \phi$. If $\mu$ is Gaussian, then [9] shows both (1.9) and (1.10) hold even if $B$ is a 2 -smooth space. Furthermore, the proof of (1.10) for 2-smooth spaces in [9] can be generalized to hold for other measures, but the assumptions required are quite special unless $\mu$ is Gaussian. Hence such results are omitted here.

Acknowledgements and additional remarks. After our paper was written, we became aware of [5] through some conversations with the author. The results of [5] provide the analogue of Theorem 1 for empirical measures on a Polish space $(S, d)$, and when combined with the contraction principle, and some additional work, it should yield a result like (perhaps equivalent to) our Theorem 1. However, if one looks at Sect. 5 of [6] where a somewhat similar project is carried out at the logarithmic level, one sees that such an attempt is likely to be longer than our direct approach. Furthermore, it is possible, to formulate Theorem 1 more generally so that the results of [5] follow immediately, and it is this we turn to now.

Theorem 1*. Let $B$ be a normed linear space and assume $E_{0}$ is a convex Polish subset of $B$ in the induced topology such that $E_{0}$ is also positively balanced, i.e. $\lambda x \in E_{0}$ whenever $x \in E_{0}$ and $0 \leqq \lambda \leqq 1$. Furthermore, assume $\mu \in \mathbb{P}, \mu\left(E_{0}\right)=1$, and $\mu$ satisfies (1.2). Let $m$ denote the mean of $\mu$, and assume $X, X_{1}, X_{2}, \ldots$ are i.i.d. with $\mu=\mathscr{L}(X)$ and $S_{n}=X_{1}+\cdots+X_{n}$. If $D$ is an open convex subset of $B$ such that $m \notin D$ and $D \cap \operatorname{dom}(\lambda) \neq \phi$, then there exists a unique point $a_{0} \in \partial D$ such that $a_{0}$ is a dominating point for $(D, \mu)$ and (1.5) holds.

The proof of Theorem $1^{*}$ is similar to that of Theorem 1 below by applying the ideas of Theorem 4.3 in [4], and hence we do not include the details. It is of interest as it readily implies the results of [5] by letting $B$ denote the vector space of finite Borel signed measures on $(S, d)$ with norm

$$
\|v\|=\sup _{f \in L(1,1)}\left|\int_{s} f d v\right| \quad(v \in B) .
$$

Here $L(a, b)$ denotes the set of functions on $S$ bounded by $a>0$ and having Lipschitz constant $b>0$. Also let $E_{0}$ denote the class of all subprobability measures on the Borel subsets of $(S, d)$. Then $E_{0}$ is Polish in the induced topology and the dual $B^{*}$ of $B$ can be viewed as the vector space of bounded 
Lipschitz functions on $S$, see [5] for details. Using this notation, Theorem $1^{*}$ applies immediately to yield the results in [5]. It also is clear that Theorem 2 has an analogue of the form in Theorem $1^{*}$, but it is not included.

Our original proof of Proposition 1 in Sect. 5 was based on a different, and more complicated method. The present form of the proof was suggested by V. Bentkus, and we are indebted to Professors F. Götze and V. Bentkus for some useful discussions on Proposition 1. The first author U. E. also thanks F. Götze for making possible a visit to the University of Bielefeld during which part of this research was completed. Finally, we thank I. Dinwoodie for his comments regarding the relation of [5] to our Theorem 1. They resulted in Theorem 1*.

\section{Some Lemmas}

If $\mu \in \mathbb{P}$ satisfies (1.2), then the mapping $\psi: B^{*} \rightarrow B$ defined by

$$
\psi(g)=\int_{B} x e^{g(x)} d \mu(x) / \hat{\mu}(g), \quad g \in B^{*}
$$

is weak-star sequentially continuous, and the right hand term exists as a Bochner integral. If $\mu, v \in \mathbb{P}$ we define $k(v \mid \mu)$ to be the Kullback-Leibler information of $v$ relative to $\mu$, which is given by:

$$
k(v \mid \mu)= \begin{cases}\int_{B} \log \frac{d v}{d \mu} d v & \text { if } v \ll \mu \text { and } \int_{B}\left|\log \frac{d v}{d \mu}\right| d v<\infty, \\ +\infty & \text { otherwise }\end{cases}
$$

Our first lemma is mentioned in [1], and here we include a complete proof.

Lemma 2.1. If $\mu \in \mathbb{P}$ is mean zero, then $\lambda(0)=0$ and $\lambda(\cdot)$ is strictly convex on $\operatorname{dom}(\lambda)$. Hence if $B=\mathbb{R}$, we have $\lambda(\cdot)$ strictly increasing on $[0, \infty) \cap$ $\operatorname{dom}(\lambda)$.

Proof. From Theorem 5.2 of [6] we have

$$
\lambda(x)=\inf \left\{k(v \mid \mu): v \in \mathbb{P} \text { and } \int_{B} y d v(y)=x\right\},
$$

and by Theorem 2.1 of [2], the lower semi-continuity of $k(\cdot \mid \mu)$, and Lemma 5.1 of [6], if $\lambda(x)<\infty$ there exists a unique $v_{x} \in \mathbb{P}$ with $\int_{B} y d v_{x}(y)=x$ satisfying

$$
\lambda(x)=k\left(v_{x} \mid \mu\right) .
$$

Since $k(v \mid \mu)=\int_{B} \log \frac{d v}{d \mu} d v=\int_{B} \frac{d v}{d \mu} \log \frac{d v}{d \mu} d \mu$, and the function $t \log t$ is strictly convex on $(0, \infty)$, it follows easily that $k(v \mid \mu)$ is strictly convex in $v$ on the set where $k(v \mid \mu)<\infty$. Hence if $x_{1}, x_{2} \in \operatorname{dom}(\lambda)$ with $x_{1} \neq x_{2}$, then $v_{x_{1}}$ and $v_{x_{2}}$ given as in (2.4) are distinct and for $0<\alpha<1$ we have

$$
\begin{aligned}
\lambda\left(\alpha x_{1}+(1-\alpha) x_{2}\right) & =k\left(v_{\alpha x_{1}+(1-\alpha) x_{2}} \mid \mu\right) \\
& \leqq k\left(\alpha v_{x_{1}}+(1-\alpha) v_{x_{2}} \mid \mu\right) \\
& <\alpha k\left(v_{x_{1}} \mid \mu\right)+(1-\alpha) k\left(v_{x_{2}} \mid \mu\right) \\
& =\alpha \lambda\left(x_{1}\right)+(1-\alpha) \lambda\left(x_{2}\right),
\end{aligned}
$$


where the first inequality holds because of (2.3) and the definition of $v_{x}$, and the strict inequality follows from strict convexity of $k(\cdot \mid \mu)$. Hence $\lambda$ is strictly convex on $\operatorname{dom}(\lambda)$.

The next lemma is given in [3]. Remark 3 following Theorem 1 explains the notation.

Lemma 2.2. $\psi\left(B^{*}\right) \subseteq \operatorname{dom}(\lambda) \subseteq \overline{\psi\left(B^{*}\right)}$ and $\overline{\operatorname{co}(\operatorname{supp} \mu)}=\overline{\psi\left(B^{*}\right)}$

Lemma 2.3. If $D$ is open, convex, $0 \notin \bar{D}$, and $D \cap \operatorname{dom}(\lambda) \neq \phi$, then there exists a unique point $a_{0} \in \partial D$ such that $0<\lambda\left(a_{0}\right)<\infty$ and

$$
\lambda\left(a_{0}\right)=\inf _{x \in D} \lambda(x)=\inf _{x \in \bar{D}} \lambda(x) .
$$

Remark. The conclusion of Lemma 2.3 is given in [1], but with only a few lines of explanation. We include a proof since the details are useful in what follows.

Proof of Lemma 2.3. If $D$ is open and convex, then by the proof of Theorems 3.2 and 3.3 in [3] it follows that

$$
\inf _{x \in D} \lambda(x)=\inf _{x \in \bar{D}} \lambda(x)
$$

Hence we need only show the left-hand equality in (2.5), and that $a_{0} \in \partial D$ exists and is unique.

Now $D \cap \operatorname{dom}(\lambda) \neq \phi$ implies $\inf _{x \in D} \lambda(x)<\infty$, and since $0 \notin \bar{D}$ there exists an open ball $U$ centered at zero such that $U \cap D=\phi$. Since $\lambda(\cdot)$ has compact level sets (recall we are assuming (1.2)), there exists a sequence $\left\{x_{j}\right\} \subseteq D \cap \operatorname{dom}(\lambda)$ such that $\lim _{j} x_{j}=x_{0}, \lim _{j} \lambda\left(x_{j}\right)=\inf _{x \in D} \lambda(x)$. Hence $x_{0} \in \bar{D}$ and since $\lambda(x)=0 \Leftrightarrow x=0$ (recall the mean of $\mu$ is zero) we have

$$
0<\lambda\left(x_{0}\right) \leqq \underline{\lim } \lambda\left(x_{j}\right)
$$

as $\lambda$ is lower semi-continuous on $B$. Thus $0<\inf _{x \in D} \lambda(x)<\infty, x_{0} \in \operatorname{dom}(\lambda)$, and since $\lambda(\cdot)$ is strictly convex on the ray $\left[0, x_{0}\right]=\left\{x=t x_{0}: 0 \leqq t \leqq 1\right\}$ with $\lambda(x) \geqq 0, \lambda(0)=0$, we have $\lambda(\cdot)$ strictly increasing and positive on $\left[0, x_{0}\right]$. Thus $a_{0}=x_{0} \in \partial D$, and (2.5) holds.

To see $a_{0}$ is unique, assume $a_{1}, a_{2} \in \partial D$ and $\lambda\left(a_{1}\right)=\lambda\left(a_{2}\right)=\inf _{x \in D} \lambda(x)=$ $\inf _{x \in \bar{D}} \lambda(x)$. Then $\lambda(\cdot)$ strictly convex on the ray $\left[a_{1}, a_{2}\right]$ and $a_{1} \neq a_{2}$

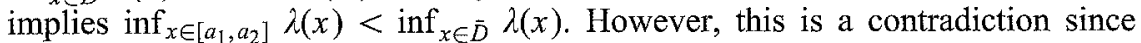
$\left[a_{1}, a_{2}\right] \subseteq \bar{D}$. Thus $a_{1}=a_{2}$ and $a_{0}$ is unique, and the lemma is proven.

Next observe that since $D$ is open and convex and by the convexity of the set $\left\{x: \lambda(x) \leqq \lambda\left(a_{0}\right)\right\}$ we can use the Hahn-Banach theorem to find an $f \in B^{*}$ such that

$$
\sup _{\left\{x: \lambda(x) \leqq \lambda\left(a_{0}\right)\right\}} f(x)=f\left(a_{0}\right)=\inf _{x \in D} f(x) .
$$


For such an $f \in B^{*}$, we define for $-\infty<t<\infty$

$$
b_{t}=\psi(t f)
$$

where $\psi$ is given by (2.1).

Lemma 2.4. Given $f$ satisfying (2.6) and $a_{0}$ as in Lemma 2.3, we have

$$
\begin{aligned}
& \text { (i) } \lambda\left(b_{t}\right)=t f\left(b_{t}\right)-\log \hat{\mu}(t f) \text {. } \\
& \text { (ii) If } \mu^{f}=\mu \text {-distribution of } f \text { on }(-\infty, \infty) \text {, then } \lambda_{\mu f}\left(f\left(b_{t}\right)\right)=\lambda\left(b_{t}\right) \text {. } \\
& \text { (iii) } \operatorname{dom}\left(\lambda_{\mu f}\right) \cap\left(f\left(a_{0}\right), \infty\right) \neq \phi \text {. }
\end{aligned}
$$

Proof. To prove (i), let $d v_{b_{t}}=\frac{e^{t f(\cdot)}}{\hat{\mu}(t f)} d \mu$. Then $\int_{B} y d v_{b_{t}}(y)=b_{t}$, so $(2.3)$ implies

$$
\begin{aligned}
\lambda\left(b_{t}\right) \leqq k\left(v_{b_{t}} \mid \mu\right) & =\int_{B}[t f(\cdot)-\log \hat{\mu}(t f)] d v_{b_{t}} \\
& =t f\left(b_{t}\right)-\log \hat{\mu}(t f) .
\end{aligned}
$$

On the other hand,

$$
\lambda\left(b_{t}\right)=\sup _{g \in B^{*}}\left[g\left(b_{t}\right)-\log \hat{\mu}(g)\right],
$$

so setting $g=t f$ we have

$$
\lambda\left(b_{t}\right) \geqq t f\left(b_{t}\right)-\log \hat{\mu}(t f) .
$$

Combining (2.9) and (2.11) implies (i).

Applying $f$ to $b_{t}=\psi(t f)$, it follows from the argument to establish (i) that if $d v_{f\left(b_{t}\right)}=\frac{e^{t u}}{\hat{\mu}^{f}(t)} d \mu^{f}$, then

$$
\begin{aligned}
\lambda_{\mu}\left(f\left(b_{t}\right)\right) & =k\left(v_{f\left(b_{t}\right)} \mid \mu^{f}\right) \\
& =t \int_{\mathbb{R}} u d v_{f\left(b_{t}\right)}(u)-\log \hat{\mu}^{f}(t) \\
& =t f\left(b_{t}\right)-\log \hat{\mu}^{f}(t) .
\end{aligned}
$$

Thus (2.8-ii) holds since $\hat{\mu}^{f}(t)=\hat{\mu}(t f)$.

To verify (iii) observe that (supp $\left.\mu^{f}\right) \cap\left(f\left(a_{0}\right), \infty\right) \neq \emptyset$, and apply Lemma 2.2 to $\mu^{f}$. The non-empty intersection follows since (supp $\left.\mu\right) \cap \tilde{D} \neq \phi$, where $\tilde{D}=\left\{x: f(x)>f\left(a_{0}\right)\right\}$. To see this note that $(\operatorname{supp} \mu) \cap \tilde{D}=\phi$ is impossible, since it would imply $\overline{c o(\operatorname{supp} \mu)} \cap \tilde{D}=\emptyset$. In view of Lemma 2.2 , this would contradict our assumption $\operatorname{dom}(\lambda) \cap D \neq \emptyset$. Thus $f(\tilde{D}) \cap \operatorname{supp} \mu^{f} \neq \emptyset$, and since $f(\tilde{D})=\left(f\left(a_{0}\right), \infty\right)$, we are done. 
Lemma 2.5. $\lambda\left(a_{0}\right)=\lambda_{\mu} f\left(f\left(a_{0}\right)\right)$.

Proof. Recalling Lemma 2.1 and (2.8-iii) and using well known continuity properties of convex functions, we see that $\lambda_{\mu} f(\cdot)$ is continuous and increasing on $[0, \tau]$ for some $\tau>f\left(a_{0}\right)$. Hence

$$
\lambda_{\mu} f\left(f\left(a_{0}\right)\right)=\inf _{s>f\left(a_{0}\right)} \lambda_{j \mu} f(s) .
$$

By Lemma 2.2 applied to $\mu^{f}$ and (2.8-ii) we also have

$$
\begin{aligned}
\inf _{s>f\left(a_{0}\right)} \lambda_{\mu f}(s) & =\inf _{\psi_{\mu} f(t)>f\left(a_{0}\right)} \lambda_{\mu}\left(\psi_{\mu} f(t)\right) \\
& =\inf _{f\left(b_{t}\right)>f\left(a_{0}\right)} \lambda_{\mu} f\left(f\left(b_{t}\right)\right) \\
& =\inf _{f\left(b_{t}\right)>f\left(a_{0}\right)} \lambda\left(b_{t}\right) \\
& \geqq \lambda\left(a_{0}\right) .
\end{aligned}
$$

The above inequality holds by (2.6) since the last infimum is only taken over $t$ 's for which $f\left(b_{t}\right)>f\left(a_{0}\right)$.

On the other hand, we trivially have,

$$
\begin{aligned}
\lambda_{\mu} f\left(f\left(a_{0}\right)\right) & =\sup _{u}\left[u f\left(a_{0}\right)-\log \hat{\mu}^{f}(u)\right] \\
& =\sup _{u}\left[u f\left(a_{0}\right)-\log \hat{\mu}(u f)\right] \\
& \leqq \sup _{g \in B^{*}}\left[g\left(a_{0}\right)-\log \hat{\mu}(g)\right] \\
& =\lambda\left(a_{0}\right) .
\end{aligned}
$$

Combining (2.13)-(2.15) we obtain the assertion of Lemma 2.5.

Lemma 2.6. There exists a unique $t_{0} \in(0, \infty)$ such that

$$
\lambda_{\mu} f\left(f\left(a_{0}\right)\right)=t_{0} f\left(a_{0}\right)-\log \hat{\mu}\left(t_{0} f\right)
$$

and

$$
b_{t_{0}}=a_{0} .
$$

Proof. To prove (2.16) simply observe that the derivative of the function $\log \hat{\mu}^{f}(t)=\log \hat{\mu}(t f),-\infty<t<\infty$, is equal to $\psi_{\mu} f(t)$. It is easy to see that the latter function is strictly increasing and continuous in $t$. Also note that $\psi_{\mu}(0)=0$. Recalling (2.8-iii) and applying Lemma 2.2 to $\mu^{f}$, we readily obtain that there exists a (unique) $t_{0}>0$ such that $\psi_{\mu_{f}}\left(t_{0}\right)=f\left(a_{0}\right)$, and it is now clear that the function $t f\left(a_{0}\right)-\log \hat{\mu}^{f}(t)$ has a maximum at $t=t_{0}$.

To verify $(2.17)$, recall that $\psi_{\mu_{f}}(t)=f\left(b_{t}\right)$, whence we have,

$$
f\left(a_{0}\right)=f\left(b_{t_{0}}\right) \text {. }
$$


As in the proof of Lemma 2.4 let $\tilde{D}=\left\{x: f(x)>f\left(a_{0}\right)\right\}$, which is obviously an open convex subset of $B$. By the definition of $f$ (see (2.6)), Lemma 2.5, (2.8-ii) and (2.18) we then have,

$$
\inf _{x \in \tilde{D}} \lambda(x)=\lambda\left(a_{0}\right)=\lambda\left(b_{t_{0}}\right)
$$

which in view of Lemma 2.3 implies (2.17).

\section{Proof of Theorem 1}

If $0 \in \bar{D}$, then $a_{0}=0$ and

$$
0=\lambda(0)=\inf _{x \in D} \lambda(x)
$$

Hence taking $g \in B^{*}$ to be the zero linear function (1.4) holds, and (1.5) is trivial in this case.

If $0 \notin \bar{D}$, then by Lemma 2.3 we have that there exists a unique point $a_{0} \in \partial D$ such that (1.3) holds. Furthermore, setting $g=t_{0} f$, we get (1.4) from Lemmas 2.5 and 2.6. Next $D \subseteq\left\{x: t_{0} f(x)>t_{0} f\left(a_{0}\right)\right\}$ follows by (2.6) along with the fact that $t_{0}>0$. This shows that $a_{0}$ is the unique dominating point of $D$. Finally note that (1.5) is immediate by defining $g$ as above and using (1.4). Moreover, it easily follows from (2.17) that $E[Z]=\int_{B} x \tilde{\gamma}(d x)=b_{t_{0}}-a_{0}=0$, and Theorem 1 has been proven.

\section{Proof of Theorem 2}

Let $a_{0}$ be the unique dominating point of $(D, \mu)$ given in Theorem 1. Since $0 \notin \bar{D}$, we have $a_{0} \neq 0$, and it follows from the proof of Theorem 1 that the representation in (1.5) holds with $g=t_{0} f$, where $f$ is as in (2.6) and $t_{0}>0$. In order to prove (1.9), we only need to show that there exists $C_{1}<\infty$ such that $J_{n} \leqq C_{1} n^{-1 / 2}$ where $J_{n}$ is defined as in Theorem 1 (with $g=t_{0} f$ ). To establish this inequality, first note that $f\left(a_{0}\right)>0$ here, so $D \subseteq\left\{x: f(x)>f\left(a_{0}\right)\right\}$, and $t_{0}>0$ together imply $t_{0} f\left(x_{1}+\cdots+x_{n}\right)>0$ for all $x_{1}+\cdots+x_{n} \in n\left(D-a_{0}\right)$. Therefore, we will have $J_{n} \leqq C_{1} n^{-1 / 2}$ provided we prove

$$
E\left[\exp \left(-t_{0}\left(f\left(Z_{1}\right)+\cdots+f\left(Z_{n}\right)\right) I_{\left\{f\left(Z_{1}\right)+\cdots+f\left(Z_{n}\right) \geqq 0\right\}}\right] \leqq C_{1} n^{-1 / 2} .\right.
$$

Now (4.1) follows easily from Lemma 6 in [1] with $Y_{j}=t_{0} f\left(Z_{j}\right)$ since $Y_{1}, Y_{2}, \ldots$ are then i.i.d. centered variables with all moments, and $t_{0} f\left(Z_{j}\right)$ has a distribution which is not concentrated at a single point. To see this note that $E[f(Z)]=0$ and $P\{f(Z)>0\} \geqq \tilde{\gamma}\left(D-a_{0}\right)>0$ as $\mu(D)>0$. Thus (1.9) holds when $a_{0} \neq 0$, and it remains to verify (1.10) and (1.11).

Now (1.5) implies (1.10) provided there exists $C_{2}>0$ such that $J_{n} \geqq$ $C_{2} n^{-1 / 2}$. Since $0 \notin \bar{D}$ and $D$ contains slices near $a_{0}$ whose diameters dominate $\tau(s)=(s|\log s|)^{1 / 2}$, we have

$$
M_{s} \cap\left(D-a_{0}\right) \supseteq\left\{y+s x_{0}: y \in M_{0},\|y\| \leqq \beta \tau(s)\right\}
$$


for $x_{0}=a-a_{0}, 0 \leqq s \leqq \delta, \beta>0$, and $M_{s}$ as given in the definition prior to the statement of Theorem 2. Thus by rescaling (4.2) with $r=s t, 0 \leqq s \leqq \delta$, we have

$$
\begin{aligned}
M_{r} \cap t\left(D-a_{0}\right) & =M_{s t} \cap t\left(D-a_{0}\right)=t\left(M_{s} \cap\left(D-a_{0}\right)\right) \\
& \supseteq\left\{t\left(y+s x_{0}\right): y \in M_{0},\|y\| \leqq \beta \tau(s)\right\} \\
& =\left\{w+r x_{0}: w / t \in M_{0},\|w / t\| \leqq \beta \tau(r / t)\right\} \\
& =\left\{w+r x_{0}: w \in M_{0},\|w\| \leqq \beta t^{1 / 2}(r|\log r / t|)^{1 / 2}\right\} .
\end{aligned}
$$

Hence

$$
\begin{gathered}
t\left(D-a_{0}\right) \supseteq\left\{x=w+r x_{0}: w \in M_{0}, 0 \leqq r \leqq t \delta,\right. \\
\left.\|w\| \leqq \beta t^{1 / 2}(r|\log r / t|)^{1 / 2}\right\} .
\end{gathered}
$$

So setting $\pi_{f}(x)=f(x) / f\left(x_{0}\right)$ and noticing $x-\pi_{f}(x) x_{0} \in M_{0}$, (4.4) implies

$$
\begin{array}{r}
t\left(D-a_{0}\right) \supseteq\left\{x=\left(x-\pi_{f}(x) x_{0}\right)+\pi_{f}(x) x_{0}: 0 \leqq \pi_{f}(x) \leqq t \delta,\right. \\
\left.\left\|x-\pi_{f}(x) x_{0}\right\| \leqq \beta t^{1 / 2}\left(\left|\pi_{f}(x)\right| \log \left|\pi_{f}(x) / t\right|\right)^{1 / 2}\right\} .
\end{array}
$$

Let $T_{n}=Z_{1}+\cdots+Z_{n}$ where $Z_{1}, Z_{2}, \ldots$ are as in Theorem 1 , and set $t=n$ in (4.5). Then, for $0<A<B$ and all $n$ sufficiently large, by the definition of $J_{n}$, and (4.5) (with $t=n$ and $\theta_{n}=\left|\pi_{f}\left(T_{n}\right) \log \left(\pi_{f}\left(T_{n}\right) / n\right)\right|^{1 / 2}$ ), we get

$$
\begin{gathered}
J_{n} \geqq e^{-t_{0} B f\left(x_{0}\right)}\left(P\left\{A<\pi_{f}\left(T_{n}\right)<B,\left\|T_{n}-\pi_{f}\left(T_{n}\right) x_{0}\right\| \leqq \beta n^{1 / 2} \theta_{n}\right\}\right) \\
\geqq e^{-t_{0} B f\left(x_{0}\right)}\left(P\left\{A<\pi_{f}\left(T_{n}\right)<B,\left\|T_{n}-\pi_{f}\left(T_{n}\right) x_{0}\right\| \leqq \beta(A n|\log B / n|)^{1 / 2}\right\}\right) \\
\geqq e^{-t_{0} B f\left(x_{0}\right)}\left(P\left\{A<\pi_{f}\left(T_{n}\right)<B\right\}-P\left\{\left\|T_{n}-\pi_{f}\left(T_{n}\right) x_{0}\right\|\right.\right. \\
\left.\left.>\beta(A n|\log B / n|)^{1 / 2}\right\}\right)
\end{gathered}
$$

Applying the Berry-Esseen Theorem with $\sigma^{2}=E\left(\pi_{f}^{2}(Z)\right)$ and $G=N(0,1)$ we have

$$
\begin{aligned}
\mid P(u / \sqrt{n} & \left.<\pi_{f}\left(T_{n}\right) /(\sigma \sqrt{n})<v / \sqrt{n}\right)-P(u / \sqrt{n}<G<v / \sqrt{n}) \mid \\
& \leqq \theta E\left|\pi_{f}(Z)\right|^{3} /\left(\sigma^{3} \sqrt{n}\right)
\end{aligned}
$$

where $\theta$ is an absolute constant. Now

$$
P(u / \sqrt{n}<G<v / \sqrt{n}) \sim(v-u) /(2 \pi n)^{1 / 2} \text { as } n \rightarrow \infty,
$$

and hence if $(v-u) /(2 \pi)^{1 / 2}>2 \theta E\left|\pi_{f}(Z)\right|^{3} / \sigma^{3}$ we have

$$
P\left(u / \sqrt{n}<\pi_{f}\left(T_{n}\right) /(\sigma \sqrt{n})<v / \sqrt{n}\right) \geqq(v-u) /\left(2 n^{1 / 2}(2 \pi)^{1 / 2}\right) .
$$

Taking $A=\sigma u, B=\sigma v$ we thus have

$$
P\left(A<\pi_{f}\left(T_{n}\right)<B\right) \geqq(B-A) /\left(2 \sigma n^{1 / 2}(2 \pi)^{1 / 2}\right)
$$


for $n$ sufficiently large. Furthermore, since $T_{n} / \sqrt{n}$ is mean zero and stochastically bounded, it follows that $\left(T_{n}-\pi_{f}\left(T_{n}\right) x_{0}\right) / \sqrt{n}$ has the same properties. Now define

$$
Y_{j}=\left(Z_{j}-\pi_{f}\left(Z_{j}\right) x_{0}\right) / \sqrt{n}
$$

for $j \geqq 1$. Then (1.2) and the arguments used in Lemmas 7 and 9 in [8] imply that $E\left\|\sum_{j=1}^{n} Y_{j}\right\|=o\left((\log n)^{1 / 2}\right)$ as $n \rightarrow \infty$. Further, observe that $\sum_{j=1}^{n} E\left\|Y_{j}\right\|^{3}=O\left(n^{-1 / 2}\right)$. Thus using the Fuk-Nagaev inequality as presented in $[7$, p.338], we have

$$
\begin{aligned}
P\left(\left\|T_{n}-\pi_{f}\left(T_{n}\right) x_{0}\right\|>\right. & \left.\beta(A n(\log n-\log B))^{1 / 2}\right) \\
\leqq & 9 \cdot 2^{11} t^{-3} O\left(n^{-1 / 2}\right) \\
& +\exp \left\{-t^{2} /\left(96 E\left\|Z-\pi_{f}(Z) x_{0}\right\|^{2}\right)\right\},
\end{aligned}
$$

where $t=\beta(A(\log n-\log B))^{1 / 2}$. Thus by setting $B=2 A$ and taking $A$ sufficiently large we have that this last probability is of order $o\left(n^{-1 / 2}\right)$ as $n \rightarrow \infty$. Hence (4.6), (4.9), and (4.10) combine to imply

$$
J_{n} \geqq A e^{-2 A t_{0} f\left(x_{0}\right)} /\left(4 \sigma(2 \pi)^{1 / 2} n^{1 / 2}\right)
$$

for $n$ sufficiently large. Thus $J_{n} \geqq C_{2} n^{-1 / 2}$ for some $C_{2}>0$, and (1.10) is proven.

We now turn to the proof of (1.11). Replacing $\tau(s)$ by $s^{1 / 2}$ and using the same arguments as in the proof of (4.5), we obtain in this case,

$$
\begin{gathered}
t\left(D-a_{0}\right) \supseteq\left\{x=\left(x-\pi_{f}(x) x_{0}\right)+\pi_{f}(x) x_{0}: 0 \leqq \pi_{f}(x) \leqq t \delta\right. \\
\left\|x-\pi_{f}(x) x_{0}\right\| \leqq \beta t^{1 / 2}\left(\left|\pi_{f}(x)\right|^{1 / 2}\right\}
\end{gathered}
$$

Setting $t=n$ and arguing as in (4.6) with $\theta_{n}=\left|\pi_{f}\left(T_{n}\right)\right|^{1 / 2}$, we have for $0<$ $A_{n}<B_{n}<\delta n^{1 / 2}$ and all sufficiently large $n$,

$$
\begin{aligned}
J_{n} & \geqq e^{-t_{0} B_{n} f\left(x_{0}\right)} P\left\{A_{n}<\pi_{f}\left(T_{n}\right)<B_{n},\left\|T_{n}-\pi_{f}\left(T_{n}\right) x_{0}\right\| \leqq \beta n^{1 / 2} A_{n}\right\} \\
& \geqq e^{-t_{0} B_{n} f\left(x_{0}\right)}\left(P\left\{A_{n}<\pi_{f}\left(T_{n}\right)<B_{n}\right\}-P\left\{\left\|T_{n}-\pi_{f}\left(T_{n}\right) x_{0}\right\|>\beta n^{1 / 2} A_{n}\right\}\right) .
\end{aligned}
$$

Now taking $A_{n}=A(\log n)^{1 / 2}, B_{n}=2 A_{n}$, and choosing $A$ large enough, we obtain by the same arguments as in the proofs of (4.9) and (4.10),

$$
P\left\{A_{n}<\pi_{f}\left(T_{n}\right)<B_{n}\right\} \sim A(\log n)^{1 / 2} /\left(\sigma n^{1 / 2}(2 \pi)^{1 / 2}\right)
$$

and

$$
P\left\{\left\|T_{n}-\pi_{f}\left(T_{n}\right) x_{0}\right\|>\beta n^{1 / 2} A_{n}\right\}=o\left(n^{-1 / 2}\right),
$$

which in combination with the above lower bound for $J_{n}$ readily implies (1.11), and Theorem 2 has been proven. 


\section{Proof of Theorem 3}

Statement (1.10) when $B$ is a Hilbert space follows easily from the representation (1.5) and the following proposition:

Proposition 1. Let $X, X_{1}, X_{2}, \ldots$ be i.i.d. mean zero random vectors with values in a Hilbert space $H$ and such that $E\|X\|^{3}<\infty$. Then for any continuous linear operator $Q: H \rightarrow H$, any $h \in H^{*}$ such that $\sigma^{2}=E h^{2}(X)>0$ and any constant $\gamma>0$ there is an absolute constant $C>0$ such that

$$
\begin{aligned}
& \left|P\left\{\left\|Q\left(S_{n} / n\right)\right\|^{2} \leqq \gamma h\left(S_{n} / n\right)\right\}-1 / 2\right| \\
& \quad \leqq \frac{C}{\sqrt{n}}\left\{\frac{E|h(X)|^{3}}{\sigma^{3}}+\frac{\left(E\|Q(X)\|^{2}\right)^{2}}{\gamma^{2} \sigma^{2}}+\frac{E\|Q(X)\|^{3}}{\left(E\|Q(X)\|^{2}\right)^{3 / 2}}\right\} .
\end{aligned}
$$

We will prove Proposition 1 following the proof of Theorem 3 .

To prove Theorem 3 , let $a_{0}$ be the unique dominating point of $(D, \mu)$, and let $f$ be as in (2.6). Set $T_{n}=Z_{1}+\cdots+Z_{n}$, where $Z, Z_{1}, Z_{2}, \ldots$ are i.i.d. with $\mathscr{L}(Z)=\tilde{\gamma}$ as defined in Theorem 1 (with $g=t_{0} f$ ). Then we have for $0<A<B$

$$
\begin{aligned}
\int_{T_{n} / n \in D-a_{0}} e^{-t_{0} f\left(T_{n}\right)} d P \geqq & e^{-t_{0} f\left(x_{0}\right) B} P\left\{A<\pi_{f}\left(T_{n}\right)<B, \frac{T_{n}}{n} \in D-a_{0}\right\} \\
\geqq & e^{-t_{0} f\left(x_{0}\right) B}\left[P\left\{A<\pi_{f}\left(T_{n}\right)<B\right\}\right. \\
& \left.-P\left\{\pi_{f}\left(T_{n}\right)>0, \frac{T_{n}}{n} \notin D-a_{0}\right\}\right],
\end{aligned}
$$

where $\pi_{f}$ is as in (4.5). Using (4.11) with $t=n$, we further have,

$$
\begin{aligned}
& P\left\{\pi_{f}\left(T_{n}\right)>0, \frac{T_{n}}{n} \notin D-a_{0}\right\} \\
& \leqq P\left\{\pi_{f}\left(T_{n}\right)>0,\left\|Q\left(T_{n} / n\right)\right\|^{2}>\beta^{2} \pi_{f}\left(T_{n} / n\right)\right\}+P\left\{\pi_{f}\left(T_{n} / n\right)>\delta\right\} \\
& \quad \leqq\left|P\left\{\pi_{f}\left(T_{n}\right)>0\right\}-\frac{1}{2}\right|+\left|\frac{1}{2}-P\left\{\left\|Q\left(T_{n} / n\right)\right\|^{2} \leqq \beta^{2} \pi_{f}\left(T_{n} / n\right)\right\}\right| \\
& \quad+\delta^{-2} \sigma^{2} n^{-1},
\end{aligned}
$$

where $Q(x)=x-\pi_{f}(x) x_{0}, x \in H$, and $\sigma^{2}=E \pi_{f}^{2}(Z)>0$. Hence by Proposition 1 (with $h=\pi_{f}, \gamma=\beta^{2}$ ) and by the Berry-Esseen inequality we have

$$
P\left\{\pi_{f}\left(T_{n}\right)>0, \frac{T_{n}}{n} \notin D-a_{0}\right\} \leqq K n^{-1 / 2},
$$

where $K<\infty$ depends on the set $D$ and the distribution of $Z$, but is independent of $n$. Combining (5.4), (5.2), (4.9), and recalling (1.5), we then have for $0<A<B$ with $(B-A) /\left(2 \sigma(2 \pi)^{1 / 2}\right)>2 \max (K, 1)$ that (1.10) holds 
with $C_{2}=e^{-t_{0} f\left(x_{0}\right) B} \max (K, 1)$. Hence Theorem 3 will hold once we establish Proposition 1.

Proof of Proposition 1. Since $\left\{x:\|Q(x)\|^{2} \leqq \gamma h(x)\right\} \subseteq\{x: h(x) \geqq 0\}$, we have $P\left\{\left\|Q\left(S_{n} / n\right)\right\|^{2} \leqq \gamma h\left(S_{n} / n\right)\right\} \leqq P\left\{h\left(S_{n} / n\right) \geqq 0\right\}$, and hence by the BerryEsseen inequality we have an absolute constant $K_{1}$ such that

$$
P\left\{\|\left. Q\left(S_{n} / n\right)\right|^{2} \leqq \gamma h\left(S_{n} / n\right)\right\} \leqq \frac{1}{2}+\frac{K_{1}}{\sqrt{n}} \frac{E|h(X)|^{3}}{\sigma^{3}}
$$

Hence it suffices to establish a similar lower bound to obtain (5.1).

To establish the necessary bound first observe that

$$
\begin{aligned}
& P\left(\left\|Q\left(S_{n} / n\right)\right\|^{2} \leqq \gamma h\left(S_{n} / n\right)\right) \\
& =P\left(2 n^{-3 / 2} \sum_{1 \leqq i<j \leqq n}\left\langle Q\left(X_{i}\right), Q\left(X_{j}\right)\right\rangle-\gamma h\left(S_{n}\right) n^{-1 / 2} \leqq-n^{-3 / 2} \sum_{i=1}^{n}\left\|Q\left(X_{i}\right)\right\|^{2}\right) \\
& \geqq P\left(2 n^{-3 / 2} \sum_{1 \leqq i<j \leqq n}\left\langle Q\left(X_{i}\right), Q\left(X_{j}\right)\right\rangle-\gamma h\left(S_{n}\right) n^{-1 / 2} \leqq-2 E\|Q(X)\|^{2} n^{-1 / 2}\right) \\
& \quad-P\left(n^{-3 / 2} \sum_{i=1}^{n} Y_{i} \geqq E\|Q(X)\|^{2} n^{-1 / 2}\right),
\end{aligned}
$$

where $Y_{i}=\left\|Q\left(X_{i}\right)\right\|^{2}-E\left\|Q\left(X_{i}\right)\right\|^{2}, 1 \leqq i \leqq n$.

Applying Markov's inequality and the Marcinkiewicz-Zygmund inequality, we have

$$
\begin{aligned}
P\left(n^{-3 / 2} \sum_{i=1}^{n} Y_{i} \geqq E\|Q(X)\|^{2} n^{-1 / 2}\right) & \leqq E\left(\left|\sum_{i=1}^{n} Y_{i}\right|^{3 / 2}\right) /\left(n E\|Q(X)\|^{2}\right)^{3 / 2} \\
& \leqq A E\left|Y_{1}\right|^{3 / 2} n^{-1 / 2} /\left(E\|Q(X)\|^{2}\right)^{3 / 2} \\
& \leqq A(8 / n)^{1 / 2}\left(E\|Q(X)\|^{3} /\left(E\|Q(X)\|^{2}\right)^{3 / 2}\right)
\end{aligned}
$$

where the last inequality is simply the $c_{r}$-inequality. Finally note that if

$$
h_{n}(x, y)=-\langle Q(x), Q(y)\rangle+\frac{\gamma n}{2(n-1)} h(x)+\frac{\gamma n}{2(n-1)} h(y),
$$

then

$$
g_{n}(x)=E\left(h_{n}\left(X_{i}, X_{j}\right) \mid X_{i}=x\right)=\frac{\gamma n}{2(n-1)} h(x)
$$

for $i \neq j$, and

$$
\frac{\gamma}{2} h\left(S_{n} / \sqrt{n}\right)-n^{-3 / 2} \sum_{1 \leqq i<j \leqq n}\left\langle Q\left(X_{i}\right), Q\left(X_{j}\right)\right\rangle=\sum_{1 \leqq i<j \leqq n} h_{n}\left(X_{i}, X_{j}\right) .
$$


Hence

$$
\begin{aligned}
& P\left(2 n^{-3 / 2} \sum_{1 \leqq i<j \leqq n}\left\langle Q\left(X_{i}\right), Q\left(X_{j}\right)\right\rangle-\gamma h\left(S_{n} / \sqrt{n}\right) \leqq-2 E\|Q(X)\|^{2} / \sqrt{n}\right) \\
& \quad=P\left(\sum_{1 \leqq i<j \leqq n} h_{n}\left(X_{i}, X_{j}\right) \geqq n E\|Q(X)\|^{2}\right),
\end{aligned}
$$

and by Corollary 4.1 of [12] we have

$$
\begin{aligned}
& P\left(\sum_{1 \leqq i<j \leqq n} h_{n}\left(X_{i}, X_{j}\right) \geqq n E\|Q(X)\|^{2}\right) \\
& \quad \geqq \Phi\left(-n E\|Q(X)\|^{2} / \sigma_{n}\right)-\frac{K_{2}}{\sqrt{n}}\left\{\frac{E\left(|h(X)|^{3}\right)}{\sigma^{3}}+\frac{\left(E\|Q(X)\|^{2}\right)^{2}}{\gamma^{2} \sigma^{2}}\right\},
\end{aligned}
$$

where (by (1.6) in [12])

$$
\sigma_{n}^{2}=\frac{1}{4} n^{3} \gamma^{2} \sigma^{2}+\frac{1}{2} n(n-1) E\left(\left\langle Q\left(X_{1}\right), Q\left(X_{2}\right)\right\rangle^{2}\right)
$$

Now

$$
\begin{aligned}
\Phi\left(-n E\|Q(X)\|^{2} / \sigma_{n}\right) & \geqq \Phi\left(-2 E\|Q(X)\|^{2} /\left(n^{1 / 2} \gamma \sigma\right)\right) \\
& \geqq \frac{1}{2}-2 E\|Q(X)\|^{2} /\left(n^{1 / 2} \gamma \sigma\right),
\end{aligned}
$$

and since

$$
\begin{aligned}
\frac{E\|Q(X)\|^{2}}{\gamma \sigma} & \leqq \max \left(\frac{\left(E\|Q(X)\|^{2}\right)^{2}}{\gamma^{2} \sigma^{2}}, 1\right) \\
& \leqq \max \left(\frac{\left(E\|Q(X)\|^{2}\right)^{2}}{\gamma^{2} \sigma^{2}}, \frac{E\|Q(X)\|^{3}}{\left(E\|Q(X)\|^{2}\right)^{3 / 2}}\right)
\end{aligned}
$$

we have from (5.6), (5.7), (5.9), (5.10), and (5.11) that

$$
\begin{aligned}
& P\left(\left\|Q\left(S_{n} / n\right)\right\|^{2} \leqq \gamma h\left(S_{n} / n\right)\right) \\
& \quad \geqq \frac{1}{2}-C n^{-1 / 2}\left(\frac{E\left(|h(X)|^{3}\right)}{\sigma^{3}}+\frac{\left(E\left(\|Q(X)\|^{2}\right)\right)^{2}}{\gamma^{2} \sigma^{2}}+\frac{E\left(\|Q(X)\|^{3}\right)}{E\left(\|Q(X)\|^{2}\right)^{3 / 2}}\right) .
\end{aligned}
$$

Hence Proposition 1 is proven.

\section{References}

1. Bolthausen, E.: On the probability of large deviations in Banach spaces. Ann. Probab. 12, 427-435 (1984)

2. Csiszar, L.: $I$-divergence geometry of probability distributions and minimization problems. Ann. Probab. 3, 146-158 (1975)

3. de Acosta, A.: On large deviations of sums of independent random vectors, Probability in Banach Spaces V, Lecture Notes in Mathematics, vol. 1153, pp. 1-14. Berlin: Springer (1985) 
4. de Acosta, A.: Upper bounds for large deviations of dependent random vectors. Z. Wahrsch. verw. Gebiete 69, 551-565 (1985)

5. Dinwoodie, I.H.: Mesures dominantes et Théorème de Sanov. Ann. Inst. Henri Poincaré 28, 365-373 (1992)

6. Donsker, M.D., Varadhan, S.R.S.: Asymptotic evaluation of certain Markov process expectations for large time III. Comm. Pure Appl. Math. 29, 389-461 (1976)

7. Einmahl, U.: Stability results and strong invariance principles for partial sums of Banach space valued random variables. Ann. Probab. 17, 333-352 (1989)

8. Einmahl, U.: Toward a general law of the iterated logarithm in Banach space. Ann. Probab. 21, 2012-2045 (1993)

9. Kuelbs, J., Li, W.V.: Some large deviation results for Gaussian measures. Probability in Banach Spaces IX, Progress in Probab., vol. 35, pp. 251-270. Basel: Birkhäuser (1994)

10. Ney, P.: Dominating points and asymptotics of large deviations in $\mathbb{R}^{d}$. Ann. Probab. 11, $158-167$ (1983)

11. Ney, P.: Convexity and large deviations. Ann. Probab. 12, 903-906 (1984)

12. Van Zwet, W.R.: A Berry-Esseen bound for symmetric statistics. Z. Wahrsch. verw. Gebiete 66, 425-440 (1984) 